\title{
La Turquie Kamâliste : voie/voix francophone(s) pour une Turquie kémaliste
}

Tanju Inal et Mümtaz Kaya

\section{(2) OpenEdition}

Édition électronique

URL : https://journals.openedition.org/dhfles/336

DOI : $10.4000 /$ dhfles.336

ISSN : 2221-4038

Éditeur

Société Internationale pour l'Histoire du Français Langue Étrangère ou Seconde

Édition imprimée

Date de publication : 1 janvier 2007

Pagination : 241-257

ISSN : 0992-7654

Référence électronique

Tanju Inal et Mümtaz Kaya, «La Turquie Kamâliste : voie/voix francophone(s) pour une Turquie kémaliste ", Documents pour l'histoire du français langue étrangère ou seconde [En ligne], 38/39 | 2007 mis en ligne le 16 décembre 2010, consulté le 27 mai 2021. URL : http://journals.openedition.org/ dhfles/336 ; DOI : https://doi.org/10.4000/dhfles.336

Ce document a été généré automatiquement le 27 mai 2021.

(c) SIHFLES 


\title{
La Turquie Kamâliste : voie/voix francophone(s) pour une Turquie kémaliste
}

\author{
Tanju Inal et Mümtaz Kaya
}

\section{Introduction}

1 L'objectif de cette communication est de survoler le long passé de la presse française ayant vu le jour sur les territoires ottoman et turc, sous divers intitulés, tels que organes de communication d'État, organe de communication des autorités françaises ou encore organes privés. En commençant chronologiquement par l'an 1795, date à laquelle parait la première publication en français à Istanbul, nous nous attarderons sur la période s'étendant de 1934 à 1948, car c'est durant cette période que la revue intitulée La Turquie Kémaliste, champ principal de notre étude, a vu le jour.

2 Pour l'histoire de la presse française de Turquie, des recherches importantes ont été menées, citons notamment celles de Hifzı Topuz, de Korkmaz Alemdar, d'Ismet Giritli, celles de Gérard Groc, réalisées en collaboration avec Ibrahim Çağlar, et enfin celle de François Georgeon. Cependant, pour ce qui est de la revue La Turquie Kémaliste, mis à part un travail de maîtrise qui lui a été consacré, elle n'a fait, à notre connaissance, l'objet d'aucune recherche détaillée en Turquie.

3 Revue illustrée, ce qui représentait une nouveauté dans l'histoire de la presse en Turquie, nous avons eu accès à La Turquie Kémaliste, à l'université de Bilkent, grâce à la collection privée de l'éminent professeur de l'histoire turque, M. Halil Inalcık.

4 La lecture passionnante et très intéressante de cette revue, vieille de soixante-douze ans et son usage d'un français très pur, nous montre que la revue était préparée par des écrivains et des artistes de l'époque, spécialistes dans différents domaines tels que l'histoire, la littérature, l'archéologie, les arts, etc. Ceci dit, le cadre de notre étude portera plus particulièrement sur les pages éditoriales dans lesquelles ressort 
principalement la lutte menée contre la désinformation concernant la République turque fondée sur des bases kémalistes.

5 Pour le survol de la presse écrite publiée en français, et marquée de temps à autre par différents incidents (conditions techniques peu développées, entraves à la publication, confiscation du matériel d'impression, difficultés financières, etc.), nous allons nous reporter à la publication de Gérard Groc et Ibrahim Çağlar tout en faisant une lecture simultanée de celle de Hifzı Topuz.

\section{La presse francophone dans l'Empire ottoman}

6 1795, 1796, 1797 sont trois dates à retenir pour l'histoire de la presse sous l'Empire ottoman. En effet, paraissent successivement les premiers journaux en français : Le Bulletin de Nouvelles, La Gazette française d'Istanbul, suivie du Mercure Oriental et du Spectateur Oriental, paru à Izmir et qui comportait quatre pages sur les nouvelles internationales et locales. D'autres journaux dont le Smyrnéen, édité à Izmir par un Français en 1824 et le Courrier de Smyrne qui soutenait le Gouvernement ottoman contre les Russes (Topuz 1960 : 12) ont très vite disparu, car leurs lignes politiques déplaisaient tant aux Ottomans qu'aux Français.

7 Les journaux de langue française publiés à Istanbul représentaient les principales sources d'informations internationales. C'est ainsi que la langue française a commencé à s'imposer comme une langue de diffusion internationale et de politique, voire comme une " langue d'extériorisation ", à tel point que même les éditeurs anglais publiaient en français ou en éditions bilingues (Groc \& Çağlar 1985 : 14).

8 On peut donc en déduire que les débuts de la presse en Turquie étaient très francophones, et que l'usage et l'apprentissage de la langue française devenaient un passage obligé, aussi bien pour les élites ottomanes qu'étrangères. La francophonie, dominante dans tous les domaines, a nécessité la création d'établissements pour l'enseignement de la langue française. C'est vers cette période que se multiplièrent les écoles françaises. Tout le long du XIX ${ }^{e}$ siècle, des dizaines d'écoles françaises, dont le lycée de Galatasaray, s'ouvrirent à Istanbul et plus de 70 écoles françaises ouvrirent leurs portes dans différentes villes d'Anatolie ${ }^{1}$.

9 Le premier journal officiel en langue turque, paru sous le règne du Sultan Mahmut II, a été le Takvim-i Vakayi. Ses rubriques contenaient des informations locales, militaires et étrangères (Topuz 1960 : 14). Sa version française paraissait sous le titre de Moniteur Ottoman. Deux mois plus tard, le journal commença à éditer en arménien et quatre ans plus tard en grec (Topuz $1960: 15$ ).

10 Comme le soulignent G. Groc et I. Çağlar, la raison de la création d'un journal officiel par le Sultan provenait du besoin de « créer une référence unique et officielle pour ses concitoyens » et pour l'étranger (Groc \& Çağlar $1985: 9$ ).

11 Il est intéressant de voir aussi comment Reşit Galip Bey, député, perçoit la création de ce premier journal dans son article intitulé «Les Recherches nouvelles autour de l'Histoire turque ", publié dans la revue La Turquie Kémaliste $e^{2}$ :

C'est Mahmut II qui, après la suppression des Janissaires, se crut libre de ses mouvements et voulut jouer le rôle d'un réformateur [...] il avait compris la nécessité de lutter contre le fanatisme qui tenait l'esprit national hermétiquement fermé à toutes les lumières de la science [...]. Et pourtant c'est lui aussi qui avait pendant de longues années hésité à demander au Cheik-ul-İslam le «fetva » pour la 
publication d'un journal officiel hebdomadaire, et cela deux siècles après les premiers journaux publiés en Occident. En effet, Le Neuw Tidingen d'Anvers commença à paraître en 1605, La Gazette, plus tard sous le nom de Gazette de France en 1631, et le Takvim-i Vakayi de Mahmut II ne verra le jour qu'en 1831. (vol. 11, 1935) française perd de son pouvoir. La loi mettait sous contrôle strict les contenus et l'autorisation de publication de nouveaux journaux. Quant aux années 1876-1908, pendant le règne du Sultan Abdülhamid, la presse s'abstient de faire toute remarque politique et ne transmet que les actualités concernant les développements commerciaux et industriels de l'Empire. C'est donc en traversant cette période de mesures et contrôles très stricts que la presse turque et francophone fait son entrée dans le $\mathrm{XX}^{\mathrm{e}}$ siècle. 


\section{La presse francophone au début du $\mathrm{XX}$ siècle}

19 Avec l'instauration du régime républicain le 29 octobre 1923, la Turquie nouvelle arbore un visage tout neuf, fait d'anticléricalisme et de laïcité, de démocratie et de pacifisme, grâce à son fondateur, Mustafa Kemal. Maître en communication, et jugeant indispensable l'exportation du modèle kémaliste, ce dernier a recours fréquemment à toutes sortes de moyens d'information tels que les journaux, les périodiques, les émissions de radio, les conférences de presse pour faire connaître la République et les réformes engagées.

Il y a lieu de rappeler qu'au début du $\mathrm{XX}^{\mathrm{e}}$ siècle et pendant les premières années de l'époque républicaine, différentes revues bimensuelles, mensuelles, trimestrielles ou annuelles ont été publiées en turc mais aussi dans d'autres langues, dont le français, et que durant la Deuxième Guerre mondiale, la presse publiée en français visait surtout les francophones vivant à l'étranger ainsi que les étrangers et Levantins vivant en Turquie. Certains quotidiens publiaient même des suppléments en français pour souligner les développements industriels et agricoles ainsi que toutes les réformes engagées dans le pays pour atteindre un mode de vie occidental; nous citerons: L'Économiste d'Orient fondé par Reşit Safet Atabinen, L'information d'Orient, organe de diffusion de l'office commercial français de Constantinople, Le petit Journal illustré fondé par Mehmed Zeki Bey, les éditions françaises des quotidiens Cumhuriyet, Akşam, Milliyet et Tanin (La République, L'Aksham, Le Milliyet, Tanine). Et enfin, toujours dans le même esprit, sera officiellement créée sur l'initiative du Gouvernement de la jeune République turque $L a$ Turquie Kémaliste, revue publiée en français et visant à faire connaître aux Levantins, aux personnels des ambassades, voire aux francophones vivant en Turquie et à l'étranger, les réformes entreprises dans le pays ainsi que l'idéal républicain basé sur les fondements kémalistes. Il nous faut parallèlement rappeler qu'à l'étranger et notamment en France, des personnalités ayant suivi de près les réformes et le « miracle turc» ont publié, suite à leur fascination devant l'œuvre accomplie par le gouvernement d'Ankara, de nombreux ouvrages et articles concernant le redressement turc, dont à titre d'exemple Gérard Tongas, Berthe Georges-Gaullis, Édouard Herriot, Paul Gentizon et K. S. Chantitch-Chandan avec son ouvrage intitulé Le Miracle Turc ${ }^{3}$.

\section{Situation de la langue française au lendemain de la Première Guerre mondiale}

21 Avec sa ratification par un minimum de trois États, dont la Grande-Bretagne, l'Italie et le Japon, le Traité de Lausanne entra en vigueur le 6 juin 1924. À la suite des élections françaises de mai 1924, Édouard Herriot remplaça Poincaré comme Président du Conseil et fit ratifier le Traité pour qu'il devienne également effectif pour la France à partir du 27 août 1924 (Soysal 1984: 605). C'est ainsi que nous assistons à une renaissance du français, langage commun de l'élite intellectuelle, dans la vie artistique et culturelle à Istanbul grâce à certaines « associations » dont particulièrement l'Union française (1895), dont le fondateur, l'ambassadeur Constans, sera aussi la personne qui fera en sorte que le quotidien L'Istanbul, fondé en 1867 par un Anglais, devienne à partir de 1895 l'organe de défense des intérêts français. L'Istanbul se maintiendra jusqu'en 1958 tout en changeant de propriétaires et de rédacteurs. 
Toutes ces associations (françaises, grecques, suisses, bulgares, etc.) fondées par les colonies étrangères qui conservaient, dans leur monde intime, un mode de vie lié étroitement à leur identité nationale, religieuse et linguistique, entretenaient entre elles des rapports très amicaux et sociaux, par le biais du français, langue des élites.

La langue française ne prédominait pas seulement dans les associations fondées par les colonies étrangères. Elle prédominait aussi dans les milieux intellectuels fréquentés majoritairement par les Turcs comme c'était le cas notamment au sein de l'université d'Istanbul, au lycée Galatasaray où l'enseignement se faisait en français, dans les cinémas, les maisons du peuple de Eminönü, de Beyoğlu, de şişli, de Kadiköy, dans le Théâtre de la ville, l'Union de la presse, l'Association des journalistes, etc.

Lieu de retrouvailles et de rencontres des intellectuels turcs et étrangers vivant à Istanbul, la très active Union française organisait des déjeuners mensuels relatifs à "L'Amitié Française », des soirées dansantes, des concerts et elle n'omettait pas non plus d'inviter des personnalités très célèbres parmi lesquelles Antoine de Saint-Exupéry qui fit une conférence sur « la poésie du voyage aérien » le 4 novembre 1935.

Nous pouvons donc dire qu'après le Traité de Lausanne, Istanbul redevient un centre où se retrouvent les communautés étrangères communiquant entre elles en français et plus tard pendant la Deuxième Guerre mondiale, Istanbul sera, avec la capitale Ankara, le lieu de refuge de ces communautés étrangères ainsi que des journalistes étrangers. C'est à la lumière de cette "photographie " sociolinguistique qu'est née, sous la Direction générale de la Presse au ministère de l'Intérieur, La Turquie Kémaliste.

\section{La Turquie Kémaliste}

\section{Historique de la revue}

26 Dans les années 1930, la jeune République turque veut se faire connaître de manière à lutter contre les préjugés occidentaux et ceux qui n'arrivent pas à se détacher de l'exotisme oriental "exporté» pendant l'Empire ottoman, voire contre ceux qui s'effraient d'une Turquie en construction et en plein essor industriel. C'est ainsi que sur l'initiative du gouvernement, Vedat Nedim Tör, diplômé du lycée Galatasaray en 1916, francophone, fera les démarches nécessaires pour la création d'une revue, La Turquie Kamâliste, qui deviendra plus tard, avec son volume 21-22 de décembre 1937, numéro spécial consacré au II ${ }^{\text {ìme }}$ Congrès d'Histoire Turque, La Turquie Kémaliste ${ }^{4}$; revue dans laquelle la langue turque ne sera pas utilisée et où dominera l'usage de la langue française.

27 Impressionné par les photographies prises par un certain Othmar Pferschy (1898-1984) d'origine autrichienne, et notamment par l'objectivité qui en découle - ce dernier ne tombe pas dans le piège de l'Occidental « romantique » impressionné par l'orientalisme - Vedat Nedim Tör l'engagera pour travailler au service de la nouvelle revue kémaliste. Ces photographies seront d'une part publiées dans la revue mais aussi dans l'album $L a$ Turquie en image/photo, premier ouvrage diffusé par la République de Turquie dans le but de la faire connaître à l'étranger.

Que ce soit à l'intérieur ou hors de Turquie, les valeurs phares du kémalisme étaient très critiquées par certains milieux. Par les partisans du Khalifat, voire les conservateurs cléricaux d'une part, par les partisans du "romantisme nostalgique " 
craignant de voir disparaître le mysticisme oriental exporté par Farrère, Loti, d'autre part et aussi par certains milieux colonisateurs, effrayés à l'idée de voir les pays sous leur joug se soulever à l'instar de la Turquie moderne. Ces jugements contrastés persistaient encore malgré une décennie écoulée depuis la proclamation de la République. D'où la nécessité d'ajouter un autre organe officiel à tous ceux déjà présents. Le message primordial de la revue était focalisé autour de deux axes : faire connaître les valeurs de la nouvelle Turquie et lutter contre les préjugés de l'Occident.

\section{Les auteurs}

La revue réunissait des textes signés par d'éminentes personnalités dont la plupart étaient des journalistes, des députés-ministres, des écrivains, comme par exemple: Vedat Nedim Tör, directeur général de la presse, le député Bolu Falih Rifkı Atay, le professeur Muzaffer Göker, secrétaire général de la Société d'Histoire turque (1937), Asım Süreyya, Hasan Ali Yücel, le ministre de l'Instruction publique, Selim Sarper, Burhan Belge, député de Muğla, Ercümend Ekrem Talu, Reşit Galip Bey, député d'Aydın et secrétaire général de la Société pour l'Étude de l'Histoire turque (1933), et aussi des contributions venant de personnalités étrangères éminentes comme les deux conférences sur la Révolution turque, prononcées en novembre 1933 par É. Herriot ${ }^{5}$, ministre d'État français.

En tout, inclus celui de É. Herriot cité ci-dessus, trois discours ont été reproduits :

1. Les deux conférences prononcées par É. Herriot en novembre 1933 et reproduites in-extenso en 1935 par Madame Y. Sarcey.

2. Les propos tenus par Atatürk conversant avec son Excellence M. Victor Antonesca, ministre des Affaires étrangères en Roumanie $\left(n^{\circ} 18,1937\right)$, reproduits en trois langues : français, allemand, anglais.

3. Le discours du Président İnönü prononcé au $5^{\text {ème }}$ congrès du parti populaire turc, 29 mai 1939 (juin 1939, vol. 31, en anglais).

\section{Tirage, fond, forme, lecteurs}

31 La revue, dont le tirage nous est resté inconnu, était surtout illustrée de nombreuses photos de citoyens turcs (enfants, femmes et hommes de différents rangs sociaux), de villes en pleine construction, de villas, d'usines, de sites archéologiques, de musées, de mosquées, d'édifices publics, d'écoles, d'instituts, de parcs, de monuments, de statues, d'hôpitaux, de paysages, etc., afin de faire connaître le redressement matériel et moral du pays. Comme nous l'avons précisé plus haut, la revue encourageait avant toutes choses, la réflexion sur la jeune République et sur la Révolution kémaliste qui était au centre du débat politique et sociologique, malgré une forte approbation des réformes par une grande partie du peuple. D'ailleurs, elle avait pour objectif principal de faire percevoir aux Occidentaux les facettes peu connues des réformes.

S'il nous faut jeter un coup d'œil aux dates de publications et aux rubriques de la Turquie Kémaliste, nous remarquons qu'entre les années 1934-1938, elle est publiée régulièrement tous les deux mois par la Direction générale de la Presse du ministère de l'Intérieur. À partir de 1938, nous rencontrons des intervalles entre les publications ; souvent deux volumes sont publiés en même temps, mais à partir de 1939, nous remarquons des intervalles considérables, par exemple, une des revues comprenant les 
volumes 32 à 40, voire la période d'août 1939 à décembre 1940. Bien sûr, nous pouvons deviner que cela est dû à la Deuxième Guerre mondiale. De même, le volume 47 paraît en 1943, alors que le volume 48 ne voit le jour qu'en décembre 1947. Le dernier volume, le $\mathrm{n}^{\circ} 49$, sera publié en mars 1948.

À travers les lignes de certains articles, nous pouvons comprendre que la revue était destinée non seulement aux institutions (ambassades, consulats, etc.) et colonies étrangères se trouvant en Turquie, notamment à Istanbul et dont, nous l'avons évoqué plus haut, le langage commun était le français, mais aussi aux pays qui s'affrontaient pendant la Deuxième Guerre mondiale. Il est intéressant de souligner que la jeune République de Turquie avait choisi le français pour faire la propagande de son idéal républicain dans le Bassin méditerranéen et dans les pays balkaniques. Nous retrouvons quelques textes rédigés en allemand et anglais, mais ils sont très peu nombreux et n'apparaissent qu'à partir de la période marquée par la Seconde Guerre mondiale.

Après la période d'absence de quatre ans évoquée ci-dessus, Nedim Veysel İlkin, nouveau Directeur général de la Presse consacre la page éditoriale du volume 48 de décembre 1947 à l'annonce de la reprise de la publication de la revue et, à travers les lignes de cet article intitulé «Continuation», nous apprenons que la revue n'était pas seulement destinée à l'élite francophone vivant en Turquie, mais aussi en Europe :

La Turquie Kémaliste, revue si appréciée et recherchée jadis par ses lecteurs, avait provisoirement cessé de paraître par suite de multiples difficultés causées par la nouvelle guerre mondiale dont notre pays, bien qu'épargné par ses répercussions immédiates, n'en a pas moins ressenti le choc. Toutefois, son absence (l'absence de la revue), n'a pas tardé à se faire remarquer dans le monde de ses habitués et de ses amis, qui l'entouraient de leur chaleureuse estime. Aussi, le Département de la Presse fut-il, bien souvent, l'objet, parfois même de pays très lointains, de demandes de renseignements sur l'éventualité de sa réapparition, signes heureux dont nous devions tenir compte. La guerre, en effet, était terminée ; mais hélas, laissant derrière elle un monde fatigué, meurtri et ravagé; des conséquences inéluctables et des répercussions imprévisibles qui devaient continuer à peser lourdement sur le destin des peuples et sur l'existence des individus. ( $n^{\circ} 48$, décembre 1947)

Des conséquences, en effet il en naîtra beaucoup après cette guerre tragique, dont l'une qui se fera sentir sur La Turquie Kémaliste : l'hégémonie de la langue anglaise dans le monde entier. Effectivement, le volume suivant paraitra en mars 1948 et sera le dernier volume de cette revue. Il serait intéressant de s'attarder quelque peu sur les rubriques de la revue pour mieux comprendre ce contre quoi la jeune Turquie républicaine a engagé la lutte.

\section{Rubriques de la revue}

La présentation de la revue suit un modèle bien tracé qui s'enrichit d'autres rubriques chemin faisant. Elle est toujours constituée d'une page introductive dans laquelle ressortent en général les échos des principes kémalistes ainsi que le cri des républicains turcs luttant contre les préjugés des Occidentaux vis-à-vis de la jeune République turque. Viennent ensuite les rubriques concernant l'histoire turque, les développements industriels, les réformes éducatives, la modernisation de l'agriculture, l'archéologie, les arts, les lettres, et aussi des traductions de quelques passages d'œuvres littéraires afin de faire connaître la littérature turque. 

d'Histoire » est consacrée à faire connaître Ankara, nouvelle capitale du pays, et est intitulée "Ankara Construit ». C'est une rubrique qui semblerait répondre aux critiques - s'élevant aussi bien en Turquie qu'au sein des étrangers passionnés par l'exotisme oriental d'Istanbul - qui manifestent leur désapprobation par rapport au choix d'Ankara comme nouvelle capitale. Ankara y est montrée comme une ville en pleine construction de villas modernes, de résidences pour les représentants étrangers, voire d'ambassades et de consulats.

Une autre rubrique intéressante est celle qui s'ajoute pour la première fois à partir du volume 18 (avril 1937) et qui sera présente dans toutes les autres publications. Intitulée "L'enfant Turc ", elle est illustrée de différents "visages d'enfants turcs », souriants et mignons. Les images ne sont accompagnées d'aucun commentaire ni de textes. Cette rubrique, ajoutée chemin faisant, semble avoir été destinée à vouloir montrer l'image moderne de la nouvelle génération turque, et de détruire l'image du «Turc» « enturbanné », « enfezé », « féroce », «brutal », « impérialiste », «barbare », envahisseur ", " assiégeant "; image qui fut véhiculée pendant des siècles par le biais d'écrivains renommés et qui perdure, malheureusement de nos jours, sous forme de « virus anti-turcs $»^{6}$.

\section{Préjugés/Désinformations}

La lutte primordiale menée par cette revue est celle engagée contre la désinformation et contre « les romantiques » vivant encore à travers les images de l'Empire ottoman et contre les «entêtés » n'admettant pas les valeurs de la République turque. Dans cette optique, il est compréhensible que la page éditoriale du premier volume de la revue, rédigée par Vedat Nedim Tör, soit consacrée à cette désinformation, et comme nous le montrerons à travers des exemples, cette lutte n'a pas été facile. Ainsi, selon V. N. Tör, «l'intellectuel occidental s'est montré, la plupart du temps, incapable de comprendre les pays restés en dehors du cadre de sa propre civilisation» (1934 vol. 1, p. 1), et de continuer: "l'intellectuel occidental, qui autrefois, regardait l'Empire ottoman à travers un prisme déformant, commence maintenant à étudier la Turquie d'Atatürk avec un regard plus libéré et un cerveau plus lucide» (vol. 1, p. 1). Pourtant, il ne peut se retenir d'apporter une critique qui se fait entendre à travers la contribution qu'il « se permet d'apporter » afin de « parfaire » cette évolution « lucide» :

Tout intellectuel désireux d'étudier la Turquie et d'écrire sur elle doit :

S'habituer à considérer la Turquie comme un État intégralement indépendant ;

Venir voir la Turquie et l'étudier sur place ;

Se préserver de l'influence des opinions intéressées des capitalistes étrangers et des milieux levantins ;

Affranchir son esprit des préjugés et des microbes néfastes d'un romantisme morbide et d'un faux esprit du pittoresque et du sensationnel que l'on s'entêtait à nous appliquer ;

Et enfin, ce qui est le plus important,

Pensant que la République turque a été l'héritière d'un pays ruiné et arriéré (l'ancien Empire ottoman), ne la comparer qu'à elle-même et estimer ainsi à leur juste valeur les élans de progrès manifestés et réalisés par elle depuis 1923. (vol. 1, p. 1)

Les volumes 5 et 6 reprennent de nouveau ce thème de la désinformation, mais cette fois les critiques apportées par les plumes de Burhan Belge et Ercümend Ekrem Talu 
sont un peu plus violentes. Ainsi, dans la page éditoriale du volume 5, intitulé «ILS DISENT QUE... », Burhan Belge fait un compte rendu de ce qui a été écrit par des plumes étrangères. Après avoir dressé une liste résumant les jugements favorables des écrivains ayant compris l'esprit de l'idéal républicain turc, il résume comme suit les jugements défavorables de ceux qu'il appelle « nos détracteurs » et qu'il qualifie d'« une poignée de malades atteints d'un romantisme qui, dans leur propre pays, est en voie de liquidation ». Selon lui :

Les milieux cléricaux, n'approuvant pas tout ce qui a été réalisé sur le terrain de la laïcité, profitent de toute occasion pour (nous) attaquer.

Certains de ces écrivains « détracteurs » craignent que l'émancipation turque sur le terrain politique et économique ne soit connue dans les pays qu'ils tiennent sous leur joug, d'autres, influencés par les récits «romantiques», craignent que l'« orientalisme exporté » disparaisse à jamais. (vol. 5, p. 1)

41 "Au siècle dernier", continue-t-il, cette poignée de malades "essayaient de venir guérir leur mal sur les rivages de la Méditerranée, dans cet Orient qui leur offrait le spectacle d'une foire perpétuelle. L'Empire ottoman était l'élément le plus varié de cette foire. Eyup a suffi à peine à remplir l'existence d'un Loti... » (vol. 5, p. 1).

Ercümend Ekrem Talu, dans la page éditoriale du volume 6, va dans le même sens que Burhan Belge et semble compléter l'article de ce dernier. Ainsi, dans son article intitulé "Oculos Habent, Sed... ", il attaque de nouveau ces romantiques qui n'ont rien compris à l'idéal kémaliste :

Ajoutons aussi qu'une partie de ces confrères étrangers portent encore - on ne sait au juste pourquoi - la mentalité de Loti et de Claude Farrère. Ils ont, tous, une littérature standard: la littérature éplorée qui gémit sur la disparition de l'«Orient ». Pour un peu, on les prendrait pour des pleureurs à solde dont le métier exige qu'ils arrosent de leurs larmes de crocodile le fez, le tcharchaf, la fenêtre à jalousies, le couvent de derviches, les chiens errants et les quelques fainéants apathiques à la cervelle creuse qui passaient leur vie à flâner et à bâiller dans les coins des cafés.

À chaque pas de plus que nous faisons sur le chemin du progrès et de la civilisation, éclate la clameur désapprobatrice de ces êtres bizarres. Et quand on leur en demande la cause ils ne trouvent rien de mieux que de répondre :

Que voulez-vous? Nous voulons servir à nos lecteurs ce qu'ils aiment; et ce qu'ils aiment est le charme, qui se perd de plus en plus, de l'« Orient ». (vol. 6, p. 1)

Nous retrouvons la même évolution de style chez Vedat Nedim Tör dans la page éditoriale du volume 8 contre "les romantiques", à la recherche d'un "Orient" disparu à jamais dans la Turquie républicaine en pleine construction :

Certains des étrangers venant visiter la Turquie nouvelle arrivent munis, comme de vieux Baedecker, de critères ne valant que pour la Turquie de la Monarchie et le Khalifat. Mais, voir la Turquie nouvelle avec les yeux de Pierre Loti équivaut à se regarder dans un miroir déformant. [...]

Savez-vous ce qu'un reporter photographe universellement connu, venu cette année (1935) à Ankara y a trouvé d'intéressant et de sensationnel ? Un paysan monté sur un âne, une vieille boutique, une chaumière menaçant ruine et un homme déguenillé.

Les écoles et les instituts, les hôpitaux, les édifices publics, les villas, les aqueducs, les parcs, les monuments, les statues de cette ville toute neuve créée sur un rythme fiévreux au milieu d'une nature rebelle n'ont su pénétrer à travers la cuirasse des préjugés de cet homme qui est également demeuré indifférent à l'essor de l'esprit nouveau qui palpite ici.

Semblable à un somnambule qui cherche la lune, il n'a cherché à Ankara que le pittoresque oriental, la sensation orientale. Et il n'a pas omis de nous dire avec un 
naïf orgueil : « Toutes ces nouveautés-là nous les possédons aussi chez nous ! (vol.

8, p. 1)

44 plume de Selim E. Sarper. Deux pages éditoriales des huit derniers volumes parus entre avril 1941 et mars 1948 sont consacrées de nouveau à ce sujet. Ce qui est intéressant, c'est que ce sont des volumes publiés pendant et après la Deuxième Guerre mondiale à laquelle la Turquie n'avait pas participé et qui semble pourtant, à travers ces articles, encore sujette à la désinformation. A travers les lignes de la page éditoriale du volume 42, avril 1941, intitulée "Les journalistes étrangers en Turquie », nous comprenons qu'une majorité des journalistes étrangers ont choisi la Turquie en cette période de guerre pour exercer leur profession :

Au cours de l'année écoulée, le nombre des journalistes étrangers séjournant dans notre pays n'a cessé d'augmenter de façon sensible [...] les renseignements transmis par les journalistes résidant en Turquie, à peu près à tous les journaux du monde, ainsi qu'à la majeure partie des Agences d'Information et des postes radiophoniques sont envoyés de façon à peu près continue. Ces renseignements sont-ils pourtant conformes à la réalité ? [...] Il va de soi, que nous nous efforçons de suivre avec un très vif intérêt les informations, qu'envoient chez eux, sur la Turquie, ces hôtes, auxquels, nous ouvrons, sans restriction, les portes de notre pays et de nos administrations. C'est ainsi qu'en suivant de près ces informations, on arrive parfois à des constatations fort curieuses. [...] Si l'on établit une comparaison entre les répercussions qu'amènent un discours, une déclaration, ou même une nouvelle radiodiffusée ou parue dans divers journaux, on est frappé par les notables différences qui existent entre les publications. (vol. 42, 1941)

Enfin, en terminant, évoquons le titre «Cancans » de l'article de Selim Sarper paru dans le $n^{\circ} 45$ et publié en octobre 1941. Rien que le titre, montre que la situation n'a guère évolué au sujet de la désinformation et que La Turquie Kémaliste qui se voulait, par le biais du français, l'organe de diffusion d'une Turquie républicaine s'étant affranchie de tous les principes traditionnels de l'époque ottomane, n'a pu détruire, malgré les illustrations reflétant sa résurrection et son épanouissement fondés sur des valeurs modernes, les images qui ont été attribuées au « Turc » depuis les premiers jours de son histoire.

\section{BIBLIOGRAPHIE}

GROC, Gérard et ÇAĞLAR, İbrahim (1985), La Presse française de Turquie de 1795 à nos jours : histoire et catalogue, Istanbul : Éditions Isis.

La Turquie Kamâliste, 1934-1948, vol. 1-49, Bilkent Üniversitesi Kütüphanesi, Halil İnalcık özel Koleksiyonu.

Regards sur Atatürk à l'aube du troisième millénaire (1998), Ouvrage collectif publié par le Centre Culturel Anatolie, Éditions A TA TURQUIE, octobre.

Documents pour l'histoire du français langue étrangère ou seconde, 38/39 | 2007 
SOYSAL, İsmail (1984), « Les Relations politiques turco-françaises (1921-1985) » (Türk Fransız Siyasal İlişkileri), Belleten, XLVII/188, Ankara, TTK, pp. 959-1044 ; traduit en français par Marianne Dumont, pp. 587-698.

TOPUZ, Hıfzı (1960), L'Information internationale dans la presse turque, Paris : C.E.L.S.E.

TURAN, şerafettin, Kendine Özgü Bir Yaşam ve Kişilik : Mustafa Kemal Atatürk, Ankara, Bilgi Yayınevi, şubat 2004, pp. 17 et 41.

VAHAPOĞLU, Hidayet (1997), Osmanlıdan Günümüze Azınlık ve Yabancı Okullar, M.E.B.

Yayınları : 3057, Eğitim dizisi : 17, İstanbul.

\section{ANNEXES}

Formulaire d'enquête de la revue :

À NOS LECTEURS.

1. «La Turquie Kamâliste » a pour but de vous faire connaître la Turquie nouvelle, la Turquie telle qu'elle est en réalité.

2. Pensez-vous que nous atteignons notre but? Êtes-vous convaincus que notre revue soit vraiment l'interprète de notre pensée?

3. Quelles sont les publications que vous désireriez voir encore dans « La Turquie Kamâliste » ?

4. Quelles critiques pouvez-vous nous adresser? Quelles lacunes voudriez-vous nous voir combler dans notre revue?

5. Faites-nous parvenir la liste de tous ceux - personnalités privées, amis et connaissances ou encore établissements publics et institutions de toutes sortes - à qui vous jugeriez utile de faire connaître « La Turquie Kamâliste ».

* Toutes les réponses doivent être adressées à la Direction Générale de la Presse au Ministère de l'Intérieur à Ankara.

\section{NOTES}

1. Voir à ce sujet : Hidayet Vahapoğlu, Osmanlıdan Günümüze Azınlık ve Yabancı Okullar, M.E.B. Yayınları : 3057, Eğitim dizisi : 17, İstanbul, 1997.

2. La Turquie Kamâliste, Les Recherches nouvelles autour de l'Histoire turque par Reşat Galip Bey, Bilkent Üniversitesi Kütüphanesi, Halil İnalcık özel Koleksiyonu, vol. 11, 1935.

3. Voir à ce sujet, Bige Yavuz, «Fransız Gözüyle Atatürk Devrimi Üzerine Genel Değerlendirmeler ", Belleten, CLX, Ağustos 1996, Say1 : 228'den ayribasım, Türk Tarih Kurumu basımevi, Ankara, 1996.

4. Pour les raisons de la transformation du nom Kamâl en Kemal, voir şerafettin Turan, Kendine Özgü Bir Yaşam ve Kişilik: Mustafa Kemal Atatürk, Ankara, Bilgi Yayınevi, şubat 2004, pp. 17 et 41.

5. Le traité de Lausanne entra en vigueur le 6 juin 1924, alors que le gouvernement français tardait encore à le présenter au Parlement. À la suite des élections françaises de mai 1924, Édouard Herriot, nourrissant une sympathie personnelle pour Mustafa Kemal, remplaça Poincaré comme Président du Conseil et fit ratifier le Traité, de sorte que celui-ci devint aussi effectif pour 
la France à partir du 27 août 1924 (Soysal 1984, p. 605) : "Les Relations politiques turcofrançaises (1921-1985)» par İsmail Soysal, 'Türk Fransız Siyasal İlişkileri', Belleten, XLVII/188, Ankara, TTK, 1984, pp. 959-1044 ; traduit en français par Marianne Dumont, pp. 87-698.

6. Voir à ce sujet : Kaya Mümtaz, Fransa'yı saran Türk karşıtı virüsler (La France sous l'emprise des virus anti-turcs), Frankofoni, Hacettepe Universitesi, Fransız Dili ve Edebiyatı İnceleme ve Araştırmaları Ortak kitabı n 17, pp. 161-175, Ankara, 2005.

\section{RÉSUMÉS}

La revue La Turquie Kémaliste, organe francophone publié à un rythme bien soutenu entre les années 1934-1948 et visant les lecteurs francophones en Turquie et à l'étranger, marque le début d'une propagande kémaliste et constitue un événement très important aussi bien dans l'histoire de la jeune République turque que dans la diffusion de l'idéal kémaliste. C'est en analysant minutieusement les articles de cette revue à laquelle ont contribué des journalistes, des écrivains, des universitaires, des députés et ministres que les auteurs de ce travail essayent de démontrer que cet organe francophone a surtout lutté contre la désinformation de l'Europe concernant la Turquie et a contribué à susciter une réflexion sur la jeune République et sur ses réformes engagées.

La Turquie Kémaliste, a journal regularly published in French between 1934 and 1948, composed of articles written by journalists, members of Parliament and ministers, writers and academicians, marks the start of a Kemalist propaganda and plays an important role both in the history of the young Turkish Republic and in the spreading of Kemalist ideals. The authors have analysed this publication and exemplify that it succeeded in combating disinformation and encouraging fruitful discussions on the young Republic and its ongoing reforms.

\section{INDEX}

Mots-clés : revue francophone, propagande kémaliste, République turque, histoire, réformes Keywords : Journal in French, Kemalist propaganda, Turkish Republic, history, reforms

\section{AUTEURS}

\section{TANJU INAL}

École Supérieure de Langues Étrangères Appliquées, Université de Bilkent, Ankara, Turquie

\section{MÜMTAZ KAYA}

École Supérieure de Langues Étrangères Appliquées, Université de Bilkent, Ankara, Turquie 